\title{
Composición y abundancia del zooplancton en la laguna El Morro, Isla de Margarita, Venezuela
}

\section{Composition and abundance of the zooplankton in lagoon El Morro, Margarita Island, Venezuela}

\section{William Villalba ${ }^{1}$, Brightdoom Marquez-Rojas ${ }^{2 *}$, Luis $\operatorname{Troccoli~}^{3}$, Marlene Alzolar $^{4}$ y Juan López ${ }^{5}$}

1 Escuela de Ciencias Aplicadas del Mar, Universidad de Oriente, Isla de Margarita, Venezuela.

2 Lab. Zooplancton. Instituto Oceanográfico de Venezuela, Universidad de Oriente, Cumaná-Sucre.

3 Instituto de Investigaciones Científicas- Universidad de Oriente, Venezuela.

4 Hospital Dr. Agustín Rafael Hernández, Juan Griego, Isla de Margarita, Venezuela.

5 Centro de Investigaciones Ambientales (CRIA)-UDONE.

*Autor de correspondencia.

E-mail William Villalba: wvillalbaluna@gmail.com

E-mail Brightdoom Marquez-Rojas: bmarquez2001@gmail.com

E-mail Luis Troccoli: luis.troccoli@gmail.com

E-mail Marlene Alzolar: marlenalzolar@gmail.com

E-mail Juan López: juanlopez358@hotmail.com

\section{Resumen}

Se analizó la composición y abundancia del zooplancton de la laguna El Morro en la isla de Margarita, Venezuela, durante el periodo de surgencia (marzo-mayo 2011) y de relajación (junio-agosto 2011). Las muestras fueron recolectadas en 6 estaciones de las diferentes zonas de la laguna. Se realizaron calados con una red de plancton de 333 um durante 10 minutos. Se midió in situ la temperatura, salinidad y oxígeno disuelto, además de nutrientes. Se registraron valores medios de temperatura de $29.7^{\circ} \mathrm{C}$, salinidad de 36 ups, oxígeno disuelto de $5.9 \mathrm{mg} . \mathrm{L}^{-1}$. La concentración de nutrientes fue baja (medias de $0.12 ; 0.04 ; 0.03 \mathrm{mg} . \mathrm{L}^{-1}$ para nitrato, nitrito y fosfato, respectivamente). Se detectaron diferencias significativas temporales con mayores registros en la temporada de relajación, mientras que espacialmente no mostró diferencias, a pesar que en las estaciones internas (Est. 4,5,6) se obtuvo mayor abundancia. Se identificaron quince grupos zooplanctónicos, determinándose nueve especies del grupo Copepoda, dos de Cladocera, una de Decapoda, Tunicada y Rotifera y diferentes formas larvarias de distintos taxa. Las larvas de crustáceos presentaron la abundancia media y relativa más alta (47738 ind. $\mathrm{m}^{-3}$ y $46.83 \%$, respectivamente), seguido del copépodo Oithona nana (31740 ind. $\mathrm{m}^{-3} \mathrm{y} 25.94 \%$, respectivamente) y Paracalanus quasimodo (12958 ind. $\mathrm{m}^{-3}$ y $8.47 \%$, respectivamente). Esta laguna involucra la estacionalidad surgencia-relajación como un efecto importante en la distribución y abundancia del zooplancton.

Palabras clave: Isla de Margarita; lagunas costeras; larvas de crustáceos; surgencia costera; zooplancton.

\section{Citación:}

Villalba W., B. Marquez-Rojas, L. Troccoli, M. Alzolar y J. López. 2017. Composición y abundancia del zooplancton en la laguna El Morro, Isla de Margarita, Venezuela. Revista peruana de biología 24(4): 343 - 356 (diciembre 2017). doi: http://dx.doi.org/10.15381/rpb.v24i4.14062
Información sobre los autores:

WV, MA realizaron la colecta, análisis cualitativo y cuantitativo de las muestras de zooplancton y preparación del artículo. BM revisó la data, diseño y redactó el manuscrito, LT realizó los análisis estadísticos. JL realizó las mediciones de los parámetros ambientales y análisis de nutrientes. Todos lo autores revisaron y aprobaron el manuscrito.

Los autores no incurren en conflictos de intereses.
Presentado: $\quad$ 28/05/2017

Aceptado: $\quad 06 / 06 / 2017$

Publicado online: 20/12/2017
Fuentes de financiamiento: Proyecto LOCTI, Código PL-016. Gestión ambiental para el saneamiento de la laguna costera El Morro, municipio Mariño, Isla de Margarita. 


\begin{abstract}
The composition and abundance of the zooplankton of El Morro lagoon on the island of Margarita, Venezuela, during the upwelling period (March-May 2011) and relaxation (June-August 2011) were analyzed. Samples were collected at 6 stations in different areas of the lagoon. Sampling was performed with a $333 \mu \mathrm{m}$ plankton net for 10 minutes. Water Temperature, salinity and dissolved oxygen were measured in situ, in addition to nutrients. Average water temperature values of $29.7^{\circ} \mathrm{C}$, a salinity of $36 \mathrm{ups}$, and dissolved oxygen of $5.9 \mathrm{mg} . \mathrm{L}^{-1}$ were found. The concentration of nutrients was low (averages of $0.12,0.04,0.03 \mathrm{mg}^{-\mathrm{L}^{-1}}$ for nitrate, nitrite and phosphate, respectively). Significant seasonal differences were detected with greater records in the relaxation season, whereas spatially did not show differences, although in the internal stations a greater abundance was recorded. Fifteen zooplankton groups were identified, identifying nine species of Copepoda, two of Cladocera, one of Decapoda, Tunicada and Rotifera and different larval forms of different taxa. Crustacean larvae showed the highest average and relative abundance ( 47738 ind. $\mathrm{m}^{-3}$ y $46.83 \%$, respectively), followed by copepod $\mathrm{O}$. nana (31740 ind. $\mathrm{m}^{-3}$ and $25.94 \%$, respectively) and $P$. quasimodo (12958 ind. $\mathrm{m}^{-3}$ and $8.47 \%$, respectively). This lagoon involves the emergence-relaxation seasonality as an important effect on the distribution and abundance of zooplankton.
\end{abstract}

Keywords: Margarita Island; Coastal lagoons; crustacean larvae; coastal upwelling; zooplankton.

\section{Introducción}

En las últimas décadas se ha desarrollado un interés creciente en la comunidad científica por los ambientes costeros, por cuanto conforman un amplio complejo de ecosistemas integrados; particularmente aquellos localizados en áreas tropicales (Ramírez 1996, Ferrol-Schulte et al. 2013).

Entre estos ecosistemas destacan las lagunas costeras, las cuales se definen según Yáñez-Arancibia et al. (2014) y Tavares et al. (2015) como ecosistemas muy fluctuantes caracterizados fundamentalmente por la mezcla de aguas continentales y marinas, donde la intensidad y frecuencia de esta mezcla, determina en su mayor parte los cambios estacionales de las características químicas del agua y de las poblaciones biológicas; se encuentran ubicadas en sectores con fuerte dinámica marina, sujetos a cambios rápidos en sus características tanto morfo-sedimentológicas como ambientales. Lankford (1977) plantea que son cuerpos de agua con un eje longitudinal paralelo a la costa que tiene comunicación con el mar (a través de una boca o un canal) y limitada por algún tipo de barrera física o hidrodinámica. En su porción más interna, pueden existir desembocaduras de ríos continentales y presentan canales de marea y patrones de sedimentación determinados por las corrientes internas. En promedio, la profundidad es escasa. Debido a la influencia de agua dulce y salada, constituyen ambientes con un gradiente salino que disminuye de la comunicación con el mar hacia las desembocaduras internas de los ríos.

Las lagunas son el hábitat de muchas especies de plantas y animales, sitios de hibernación de aves limícolas migratorias, y albergue de especies en peligro de extinción (Vásquez-Suárez et al. 2010). Asimismo, suelen tener elevada productividad y constituir reservorios importantes de diversidad biológica en comunidades de vertebrados e invertebrados como el zooplancton (Souza et al. 2011). El zooplancton incluye organismos que forman parte de esta comunidad solo durante una fase de su ciclo vital (meroplancton), como son las formas larvarias de crustáceos, moluscos y otras especies de invertebrados que ingresan al sistema lagunar-estuarino buscando protección hasta llegar a etapas más avanzadas de su desarrollo. Otros organis- mos desarrollan todo su ciclo vital como parte del zooplancton (holoplancton); entre los cuales se destacan los copépodos, que llegan a conformar entre 70 y $90 \%$ de la comunidad y se consideran los zoopláncteres más abundantes (Álvarez-Silva \& Torres-Alvarado 2013).

El zooplancton tiene un papel fundamental en los ecosistemas costeros, debido a la posición clave que ocupa en las tramas tróficas pelágicas como nexo entre los productores primarios y los consumidores superiores (San Martín et al. 2006). Por lo tanto, se puede aseverar que la abundancia de fitoplancton y zooplancton presente en las lagunas costeras, en gran medida dependerá de la productividad de las mismas (Vásquez-Yeomans et al. 2012). Por lo tanto, los trabajos de identificar y cuantificar al zooplancton adquieren relevancia debido a su papel en la productividad local, delimitando las rutas de transferencia energética y por ende la producción secundaria potencial de un área (Hernández-Trujillo et al. 2010).

El estudio de los mecanismos que regulan la riqueza del zooplancton en sistemas acuáticos es esencial para comprender el funcionamiento y conservación de estos ambientes (Aranguren-Riaño et al. 2011). En las lagunas costeras, la variabilidad en su abundancia y distribución se modifica estacionalmente (Longhurst y Pauly 1987), acoplándose a los diferentes escenarios hidrográficos asociados a condiciones eurihalinas o estenohalinas, las cuales determinan la detección de áreas preferenciales para la selección óptima del hábitat. En la laguna El Morro de la isla de Margarita, se han realizado ciertos estudios referente a los crustáceos (Boadas 1985), peces (Maza 1986), caracterización físico-química del agua (López 2012), metales pesados en el caracol Melongena melongena (Yepez 2012), sobre macroalgas (Valerio-González et al. 2013a) y plantas vasculares (Valerio-González et al. 2013b); sin embargo, el zooplancton de esta laguna permanece desconocido. El objetivo de este estudio fue conformar un primer inventario de las especies del zooplancton presentes en la laguna El Morro de Porlamar, Nueva Esparta (Venezuela), así como aportar datos de su abundancia y distribución relacionados con las principales características hidrológicas de la laguna. 


\section{Materiales y métodos}

Área de estudio.- La Laguna El Morro, se encuentra ubicada en la costa sureste de la isla de Margarita, Venezuela, ubicada al SE del Caribe (Fig. 1), entre las coordenadas 1057’25.52”N Y 6349'25.82”W (Boadas 1985). Tiene una longitud norte-sur de $1.8 \mathrm{~km}$ y un ancho máximo de $1.5 \mathrm{~km}$ para una superficie de $127 \mathrm{~km}^{2}$, incluyendo a los islotes Caigüiré I y Caigüiré II. Fundamentalmente presenta un clima seco, con temperatura media de $27^{\circ} \mathrm{C}$, con máximas de $37^{\circ} \mathrm{C}$ y mínimas de $18.5^{\circ} \mathrm{C}$ y la precipitación de $271.7 \mathrm{~mm}$ anuales con un patrón de distribución temporal de régimen bimodal, con valores elevados de evaporación entre los meses de marzo a mayo (Ramírez 1996). En su extremo sur esta bordeada por los cerros del Morro. Por el norte limita con una serie de edificios. Por el este con una barra arenosa que la separa del mar abierto y por el oeste tiene comunicación con el mar a través de una estrecha boca, con una profundidad entre 1.5 a $2 \mathrm{~m}$.

Este cuerpo lagunar está dividido en dos partes que tienen comunicación entre sí. La parte que desemboca en la bahía de Guaraguao conocida como Laguna Negra, mientras que la parte más interna y extensa es la llamada Laguna Blanca. Esta laguna fue declarada zona Protegida en 1992 (Bevilacqua et al. 2006).

Recolección y procesamiento de las muestras.- Debido a las características que presenta la laguna, fueron seleccionadas seis estaciones de muestreo: 3 dentro del cuerpo lagunar, con profundidades entre 1 y 3.5 metros y 3 estaciones en el canal de entrada que comunica con él área costera aledańa, con profundidades entre 1.5 y $2 \mathrm{~m}$ (Fig. 1). Se realizaron los muestreos a nivel superficial (aprox. 1 metro) entre las 8:00 y 10:30 am, entre marzo y agosto del 2011.

En cada estación se hicieron mediciones de temperatura del agua $\left({ }^{\circ} \mathrm{C}\right)$, oxígeno disuelto $(\mathrm{mg} / \mathrm{L})$ y salinidad, mediante una sonda multiparamétrica YSI 600R, acoplada a un capturador de datos YSI 650 DMS.
Las muestras de agua para los análisis de nutrientes se tomaron con una botella de Van Dorn superficialmente (aprox. 1 metro), posteriormente fueron colocados en envases de plásticos de un litro de capacidad con tapa hermética. Las muestras fueron colocadas en un recipiente con hielo y trasladadas al laboratorio de calidad ambiental del Centro Regional de Investigaciones Ambientales (CRIA). Las determinaciones de los nutrientes (nitrito, nitrato y fosfato) se realizaron siguiendo la metodología descrita según los métodos estándares de la American Public Health Association, American Water Works Association y Water Environment Federation (APHA-AWWA-WEF 1998).

En cada estación, se recolectaron muestras de zooplancton con una red estándar de $30 \mathrm{~cm}$ de diámetro de boca, largo de 90 cm y luz de malla de $300 \mu \mathrm{m}$; el volumen de agua filtrado por la red se determinó con datos de flujómetro. La red fue arrastrada durante 10 minutos a una velocidad entre 1 y 1.5 nudos. Las muestras fueron fijadas con formol a una concentración del $4 \%$, neutralizado con tetraborato de sodio.

Los análisis cualitativos y cuantitativos se llevaron a cabo utilizando un microscopio óptico y uno de disección, ambos marca Leica. Se contabilizó por lo menos el 10\% de la submuestra de volumen conocido, extraída con una pipeta Stempel (Boltovskoy 1981); el número de organismos contados se dividió entre el volumen de agua filtrada, por lo que los resultados se estandarizaron a ind. $\mathrm{m}^{-3}$.

Para la ubicación taxonómica de los organismos se utilizaron las siguientes referencias Smith (1977), Campos-Hernández y Suárez-Morales (1994), Suárez-Morales et al. (1996), Boltovskoy (1999), Esnal (1999), Razouls et al. (2005), Gutiérrez et al. (2008).

Análisis de los datos.- Luego de comprobar que no se cumplieron los supuestos del análisis paramétrico mediante la prueba de normalidad de Kolmogorov-Smirnov y homocedasticidad de Levene (Sheskin 2004), se utilizaron las pruebas no paramétrica
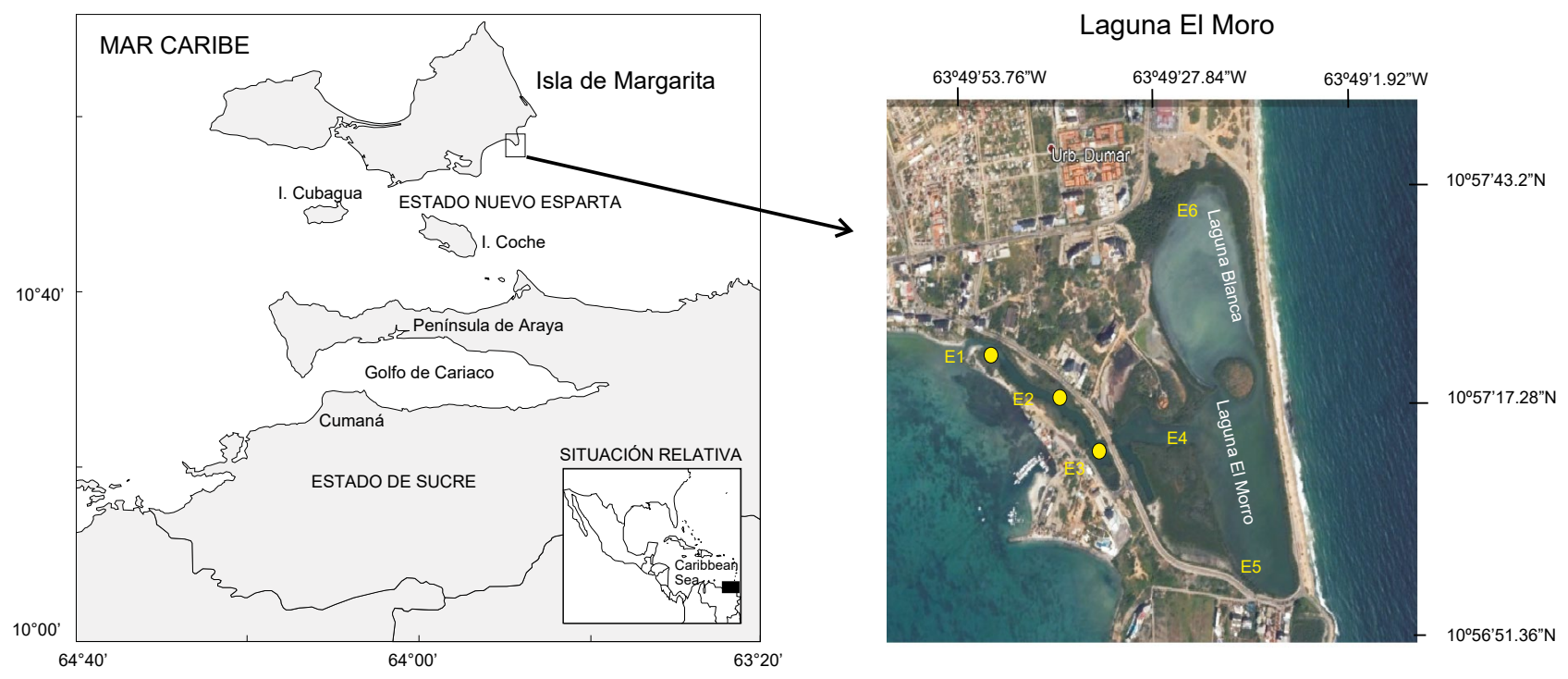

Figura 1. Ubicación de las estaciones de muestreos, en la Laguna El Morro, Isla de Margarita, Venezuela. 
de Kruskal-Wallis y el test de Mann-Whitney o Wilcoxon. La comparación de las variables ambientales y de la abundancia zooplanctónica entre los meses y estaciones se realizó con el análisis de Kruskal-Wallis. Posteriormente se procedió a dividir los meses en dos períodos, de acuerdo a la estacionalidad de la zona (Lorenzoni et al. 2013, López-Monroy \& Troccoli 2015), época de surgencia (marzo, abril y mayo) y época de relajación (junio, julio y agosto) y las estaciones también fueron divididas en dos grupos, de acuerdo con su ubicación, correspondiendo la zona externa (estaciones $1,2,3$ ) a las ubicadas en la entrada y la zona interna (estaciones 4,5,6) correspondientes al interior de la laguna. Las comparaciones de las 2 zonas y de los períodos estudiados, se realizaron con la prueba de Mann-Whitney. Se graficaron los resultados con diagramas de cajas (boxplot) de acuerdo a las recomendaciones de Boyer et al. (1997).

Se realizó un análisis de escalamiento multidimensional no métrico (MDS), sobre la distribución y abundancia de los principales grupos del zooplancton. Se efectuó la transformación de la raíz cuarta de los datos para crear una matriz de similitud de Bray-Curtis. Para visualizar las diferencias en los grupos del mesozooplancton en dos dimensiones con los datos de abundancia de cada zona y períodos estudiados.

Con las especies que aparecieron en porcentaje mayor al 10\% (Legendre \& Legendre, 1998) se realizó el análisis de similitud (ANOSIM) para probar las diferencias entre los grupos de la comunidad del zooplancton entre las zonas y períodos. La identificación de los principales grupos taxonómicos que más contribuyen a la similitud y la disimilitud entre las zonas y los períodos de estudio se realizó con la prueba de porcentaje de similitud (SIMPER), para esto se utilizó el Primer $6 \&$ Permanova (Clarke \& Gorely 2006).

Con el fin de conocer las posibles relaciones entre las especies que aparecieron en porcentaje mayor al 10\% y las variables ambientales registradas, se efectuó un análisis de correspondencia canónica (ACC). Este procedimiento permite posicionar los grupos de organismos y especies con los factores ambientales en un sistema sintetizado de coordenadas (componentes 1 y 2) (Ter Braak \& Verdonschot 1995). En todas las pruebas estadísticas, se consideró una significancia de $p=0.05$.

\section{Resultados}

Variables ambientales.- La temperatura superficial del agua osciló entre $26.7-33.2^{\circ} \mathrm{C}$, con una media de $29.7^{\circ} \mathrm{C}$. Se detectaron diferencias significativas temporales $(\mathrm{KW}=15.78$, $p<0.05$; Fig. $2 \mathrm{~A}$ ) y por período, registrando las temperaturas más altas en relajación $(\mathrm{W}=287.5, p<0.05$; Fig. $2 \mathrm{C})$. Con respecto a las estaciones, no se halló diferencias significativas $(\mathrm{KW}=9.39, p>0.05$; Fig. 2B); sin embargo, por ubicación de las estaciones, la zona interna exhibió los valores más altos ( $\mathrm{W}=$ 242.5, $p<0.05$; Fig. 2A).
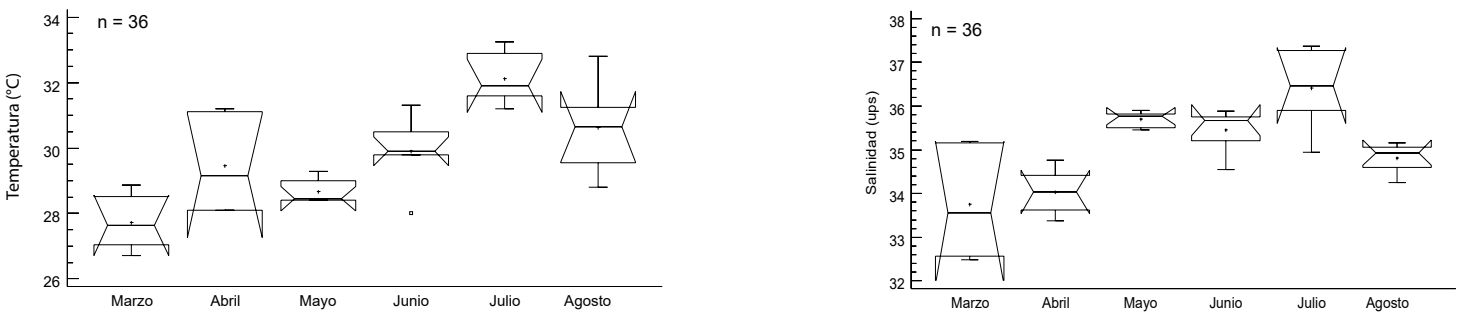

(B)

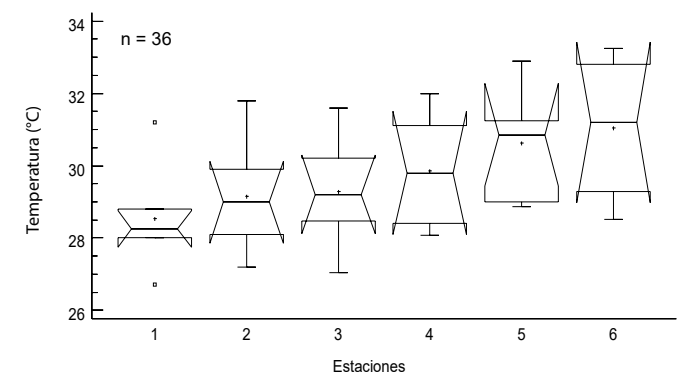

( C)

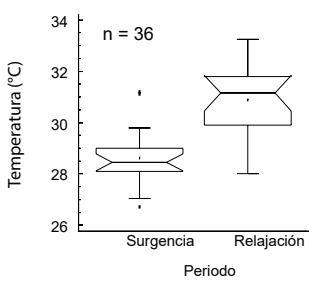

(D)

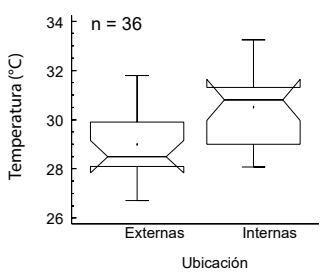

Figura 2.- $(A-D)$ Variación temporal y espacial de la temperatura $\left({ }^{\circ} \mathrm{C}\right)$ y $(E-H)$ de la salinidad (ups), en la Laguna El Morro, Isla de Margarita, Venezuela.
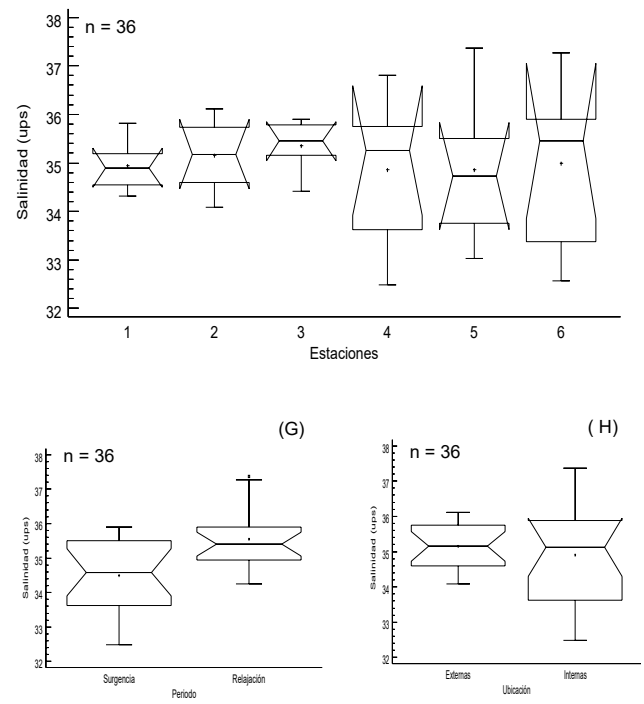
La salinidad obtuvo una media de 36 ups, con valores mínimos de 32.5 y máximos de 37.7. Esta variable mostró diferencias significativas con respecto a la temporalidad $(\mathrm{KW}=25.12, p<$ 0.05 ; Fig. $2 \mathrm{E}$ ), registrando los valores más altos en relajación (W= 238, $p<0.05$; Fig. $2 \mathrm{G}$ ), pero no se detectaron diferencias espaciales $(\mathrm{KW}=1.42 ; \mathrm{W}=150, p>0.05$; Fig. $2 \mathrm{~F}, \mathrm{H})$.

El oxígeno disuelto fluctuó entre $3.4-7.4 \mathrm{mg} . \mathrm{L}^{-1}$, con una media de $5.9 \mathrm{mg} . \mathrm{L}^{-1}$. Esta variable no presentó diferencias significativas entre los períodos y los sitios de muestreo (estaciones) (W= 206.5 y W=103.5; $p>0.05$ ). De igual manera, el nitrato tampoco mostró diferencias significativas para los dos factores estudiados $(\mathrm{W}=107.5$ y W=189.5; $p>0.05)$; esta variable mostró concentraciones entre $0.03-0.5 \mathrm{mg} . \mathrm{L}^{-1}$, con una media de $0.12 \mathrm{mg}$. $\mathrm{L}^{-1}$.

Las concentraciones de nitrito fueron bajas, con valores entre $0.001-0.3 \mathrm{mg} . \mathrm{L}^{-1}$, con una media de $0.04 \mathrm{mg} . \mathrm{L}^{-1}$. No se detectaron diferencias temporales significativas $(\mathrm{KW}=10.33, p>0.05$; Fig. 3A), mientras que por estaciones si se hallaron diferencias significativas $(\mathrm{KW}=15.65, p<0.05$; Fig. $3 \mathrm{~B})$, al igual que entre la ubicación de las estaciones ( $\mathrm{W}=270.5, p<0.05$; Fig. 3C), alcanzando las concentraciones más elevadas en la zona interna

De igual manera, las concentraciones de fosfato fueron relativamente bajas, con fluctuaciones entre $0.001-0.3 \mathrm{mg} . \mathrm{L}^{-1}$, con una media de $0.03 \mathrm{mg} . \mathrm{L}^{-1}$; no obstante, esta variable mostró diferencias temporales $(\mathrm{KW}=6.31 \mathrm{y} W=230.5, p<0.05$; Fig. $3 \mathrm{D}, \mathrm{F})$ y espaciales significativas $(\mathrm{KW}=22.37 \mathrm{y} \mathrm{W}=285.5$; $p<$
0.05), alcanzando los valores más altos en el período de relajación y en las estaciones internas (Fig. 3E, G).

Sistemática y abundancia.- Se identificaron 9 especies de la subclase Copepoda, 2 de Cladócera, una de Decapoda, de Tunicada, de Rotifera y diferentes formas larvarias de distintos taxa. Las especies identificadas se presentan en la Tabla 1.

En general, se recolectó un total de 611629 ind. $\mathrm{m}^{-3}$. La abundancia zooplanctónica osciló entre 10198 - 329700 ind.m ${ }^{-3}$, con un valor medio de 117131 ind. $\mathrm{m}^{-3}$ (Tabla 1 ).

La abundancia zooplanctónica mostró diferencias significativas temporales $(\mathrm{KW}=26.17, p<0.05$; Fig. $4 \mathrm{~A})$ y entre los períodos (W= 232, $p<0.05$; Fig. $4 \mathrm{~B})$, registrando los valores más altos $\left(164133-329700\right.$ ind. $\left.\mathrm{m}^{-3}\right)$ en relajación, específicamente en julio y agosto 2011. En el análisis MDS (Stress=0.16) se muestra mayor dispersión de la comunidad durante relajación, indicando posiblemente dos grupos separados (Fig. 4C).

Durante marzo, la abundancia del zooplancton osciló entre 1566 (Est.3) y 10866 (Est. 1) ind. $\mathrm{m}^{-3}$, con una media de 5189 ind. $\mathrm{m}^{-3}$; las larvas de crustáceos y los copépodos conformaron el $90 \%$ del total (Tabla 1; Fig. 5). En abril, la abundancia varió entre 2133 (Est.2) y 6933 (Est. 4) ind. $\mathrm{m}^{-3}$, la media fue 3878 ind. $\mathrm{m}^{-3}$; en este mes hubo una dominancia de los copépodos (75\%), principalmente Nanocalanus minor y Acartia tonsa, luego le siguieron las larvas de crustáceos con 13\% (Tabla 1; Fig.
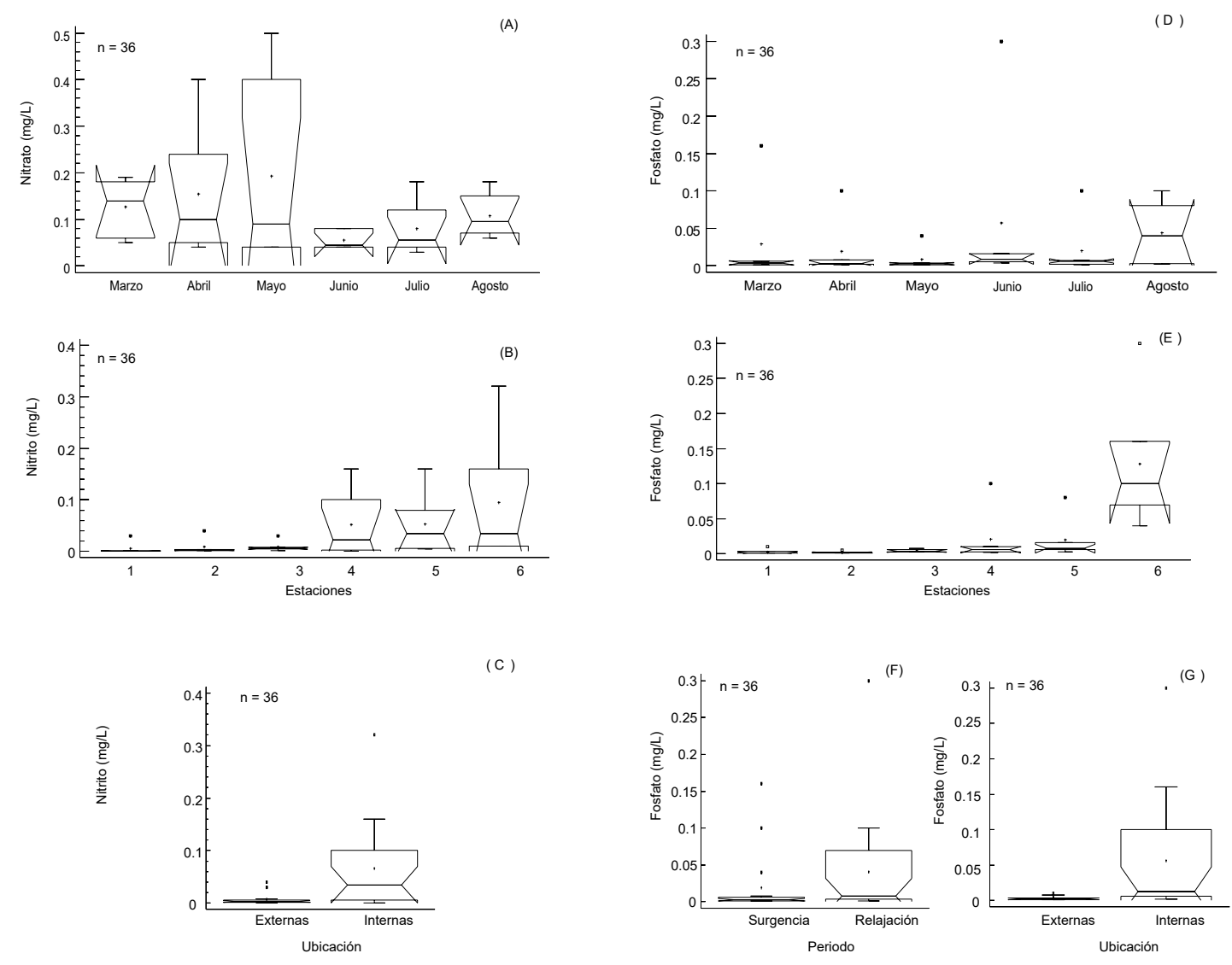

(c)
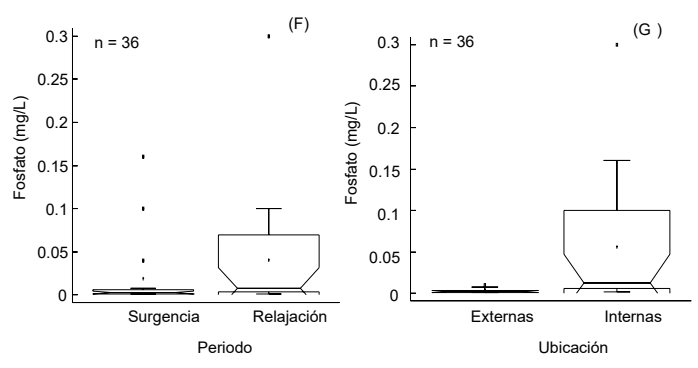

Figura 3.- $(A-D)$ Variación temporal y espacial del Nitrito $(\mathrm{mg} / \mathrm{L})$ y $(E-H)$ del Fosfato $(\mathrm{mg} / \mathrm{L})$, en la Laguna El Morro, Isla de Margarita, Venezuela. 
Tabla 1. Abundancia media (ind. $\mathrm{m}^{-3}$ ) y relativa (\%) de los organismos zooplanctónicos contabilizados en la Laguna del Morro, Isla de Margarita, Nueva Esparta, Venezuela. $(n=133)$.

\begin{tabular}{|c|c|c|c|c|c|c|c|c|c|}
\hline ORGANISMOS/MES & Mar & Abr & May & Jun & Jul & Ago & Total & Media & Abu. Relati \\
\hline \multicolumn{10}{|l|}{ RADIOLARIA } \\
\hline Radiolarios & & & & 100 & & & 100 & 100 & 0.02 \\
\hline \multicolumn{10}{|l|}{ CNIDARIA } \\
\hline Medusa & 234 & 167 & 400 & & 2566 & 100 & 3467 & 693 & 0.57 \\
\hline \multicolumn{10}{|l|}{ ANNELIDA } \\
\hline Oligoquetos & & & & 200 & 2134 & 333 & 2667 & 889 & 0.44 \\
\hline Larvas de poliquetos & 100 & & 67 & 167 & 1468 & 200 & 2002 & 400 & 0.33 \\
\hline \multicolumn{10}{|l|}{ COPEPODA } \\
\hline Acartia (Acanthacartia) tonsa Dana, 1849 & 3566 & 3100 & 1899 & 2233 & 10767 & 2000 & 23565 & 3928 & 3.85 \\
\hline Nannocalanus minor (Claus, 1863) & & 4099 & 1367 & & 6667 & 4567 & 16700 & 4175 & 2.73 \\
\hline Paracalanus quasimodo Bowman, 1971 & 834 & & & 866 & 11067 & 39067 & 51834 & 12958 & 8.47 \\
\hline Paracalanus aculeatus Giesbrecht, 1888 & & & 367 & 167 & 6166 & 3767 & 10467 & 2617 & 1.71 \\
\hline Temora turbinata (Dana, 1849) & & 200 & & 134 & 233 & 367 & 934 & 233 & 0.15 \\
\hline Oithona nana Giesbrecht, 1892 & 2033 & 234 & & 400 & 69434 & 86600 & 158701 & 31740 & 25.95 \\
\hline Oithona sp. & 2100 & 600 & 300 & 200 & 1667 & 800 & 5667 & 945 & 0.93 \\
\hline Triconia conifera (Giesbrecht, 1891) & 301 & 566 & 267 & 133 & 867 & 100 & 6234 & 1039 & 0.37 \\
\hline Euterpina acutifrons (Dana, 1847) & 666 & & 467 & & 6401 & 1100 & 8634 & 2159 & 1.41 \\
\hline Copepoditos & 3067 & 8700 & 2666 & 2799 & 4866 & 200 & 22298 & 3716 & 3.65 \\
\hline \multicolumn{10}{|l|}{ CLADOCERA } \\
\hline Moina micrura & & 167 & & 266 & 300 & 100 & 833 & 208 & 0.14 \\
\hline Penilia avirostris Dana, 1849 & 167 & & 133 & 599 & & & 899 & 300 & 0.15 \\
\hline \multicolumn{10}{|l|}{ DECAPODA } \\
\hline Belzebub faxoni (Borradaile, 1915) & & 67 & 167 & & & & 234 & 117 & 0.04 \\
\hline Nauplios de crustáceos & 15232 & 2967 & 1067 & 43500 & 200733 & 22067 & 285566 & 47594 & 46.69 \\
\hline Zoeas de crustáceos & 67 & 100 & 33 & 100 & 533 & 33 & 866 & 144 & 0.14 \\
\hline \multicolumn{10}{|l|}{ EUPHAUSIACEA } \\
\hline Eufasidos & 100 & 33 & & 67 & & & 200 & 67 & 0.03 \\
\hline \multicolumn{10}{|l|}{ OSTRACODA } \\
\hline Ostracodos & & 67 & 100 & & 66 & 67 & 300 & 75 & 0.05 \\
\hline \multicolumn{10}{|l|}{ CHAETOGNATHA } \\
\hline Sagitta spp & 100 & 399 & 66 & & 2067 & 1167 & 3799 & 760 & 0.62 \\
\hline \multicolumn{10}{|l|}{ TUNICADA } \\
\hline Oikopleura dioica Fol, 1872 & 100 & 267 & 300 & 67 & 99 & 66 & 899 & 150 & 0.15 \\
\hline \multicolumn{10}{|l|}{ VERTEBRATA } \\
\hline Huevos de peces & 467 & 833 & 167 & 401 & 266 & 1000 & 3134 & 522 & 0.51 \\
\hline Larvas de peces & 166 & 333 & 66 & 133 & 66 & 300 & 1064 & 177 & 0.17 \\
\hline \multicolumn{10}{|l|}{ NEMATODA } \\
\hline Nematodos & 400 & 100 & 99 & & 801 & 33 & 1433 & 287 & 0.23 \\
\hline \multicolumn{10}{|l|}{ TURBELLARIA } \\
\hline Turbelario & & 67 & & & 466 & 33 & 566 & 189 & 0.09 \\
\hline \multicolumn{10}{|l|}{ ROTIFERA } \\
\hline Brachionus plicatilis Müller, 1786 & & & & 1100 & & & 1100 & 1100 & 0.18 \\
\hline \multicolumn{10}{|l|}{ MEROPLANCTON } \\
\hline Larvas de cirrípedos & 167 & 500 & & & & & 667 & 334 & 0.11 \\
\hline Larvas de Equinodermos & 100 & 33 & & & & 33 & 166 & 55 & 0.03 \\
\hline Larvas de moluscos & 166 & 167 & 200 & 67 & & 33 & 633 & 127 & 0.10 \\
\hline TOTAL & 30133 & 23766 & 10198 & 53699 & 329700 & 164133 & 611629 & 117131 & 100 \\
\hline
\end{tabular}

5). Durante mayo se registró un intervalo de abundancia entre 1166 (Est. 2) y 3133 (Est. 1) ind. $\mathrm{m}^{-3}$, con una media de 1722 ind. $\mathrm{m}^{-3}$. En este mes, los copépodos constituyeron el $71 \% \mathrm{del}$ zooplancton y las especies presentes fueron $A$. tonsa y $N$. minor, otros componentes importantes fueron las larvas de crustáceos (11\%), los cladóceros (4\%) y las medusas (4\%). En junio la abundancia osciló entre 567 (Est. 3) y 4165 (Est.5) ind.m ${ }^{-3}$ (media: 1839 ind. $\mathrm{m}^{-3}$ ). Los copépodos constituyeron el 63\%, principalmente por $A$. tonsa y los copepoditos; en este mes se identificó al rotífero Brachionus plicatilis (10\%) y radiolarios
$(0.02 \%)$. En julio y agosto se detectaron los mayores registros de abundancia y diversidad de especies. La abundancia en julio varió entre 26734 (Est. 3) y 81732 (Est. 4) ind. $\mathrm{m}^{-3}$, la media fue 55417 ind. $\mathrm{m}^{-3}$; en este mes se observó la dominancia de las larvas de crustáceos (60\%), luego le siguieron los copépodos con 36\%, principalmente por Oithona nana, otros elementos importantes fueron los quetognatos (Sagitta spp.). En agosto, la abundancia registró magnitudes entre 4467 (Est. 2) y 87033 (Est. 6) ind. $\mathrm{m}^{-3}$ (media: 24722 ind. $\mathrm{m}^{-3}$ ). Los copépodos constituyeron el $84 \%$ del total, las especies dominantes fueron $O$. nana y Paracalanus 
(A)

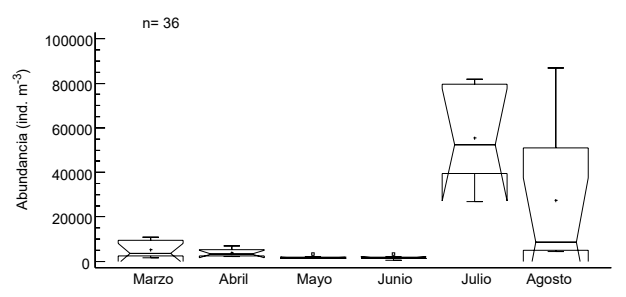

(B)

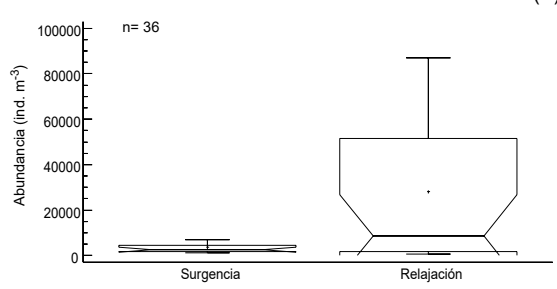

( C )

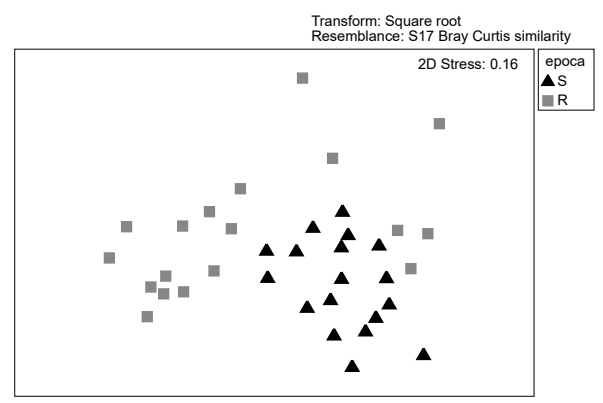

(D)

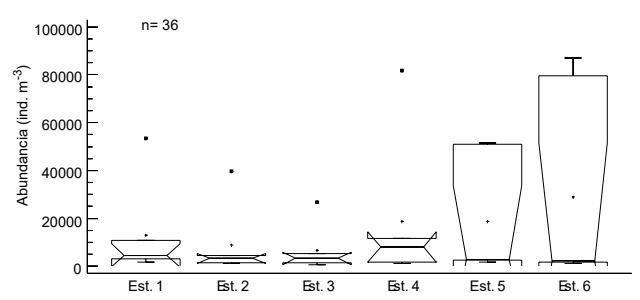

(E)

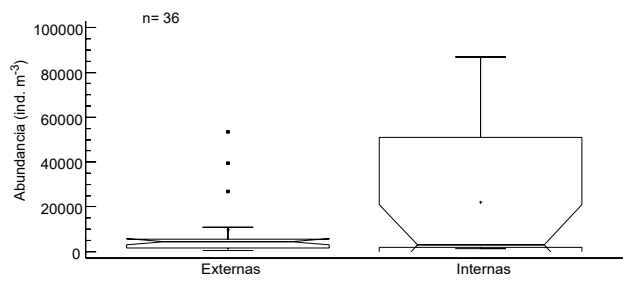

( F )

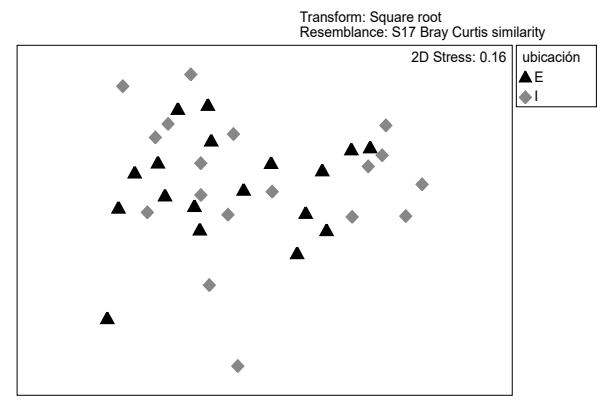

Figura 4.- Variación temporal(A, B) y estacional (D, E) de la abundancia zooplanctónica y ordenación MDS temporal y estacional de la abundancia zooplanctónica (C, F) en la laguna El Morro, Isla de Margarita, Venezuela. Por período, S: surgencia, R: relajación. Por Ubicación, E: estaciones externas y I: estaciones internas.

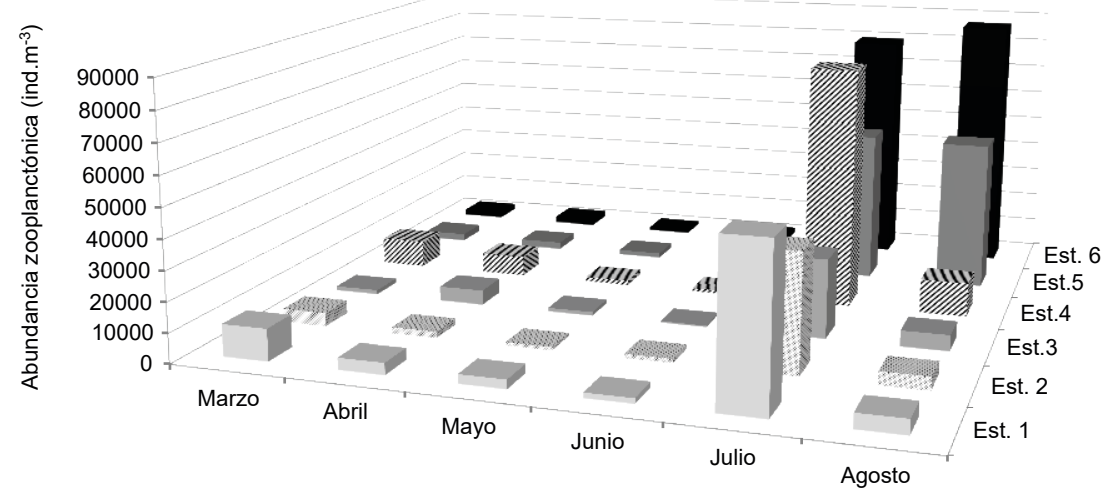

Figura 5.- Abundancia zooplanctónica (ind. $\mathrm{m}^{-3}$ ) en las seis estaciones, durante los meses de estudio en la Laguna EI Morro, Isla de Margarita, Venezuela.

quasimodo, a este le siguieron las larvas de crustáceos (13\%), en porcentajes menores pero importantes en este mes destacan los vertebrata (huevos y lavas de peces) con $2 \%$ y larvas de cirrípedos con $0.12 \%$ (Tabla 1 ).

Es importante destacar la presencia del cladócero Moina micrura, característico de agua dulce, principalmente en los meses de relajación, registrando mayor abundancia en junio y julio (266 y 300 ind. $\mathrm{m}^{-3}$, respectivamente).
La abundancia zooplanctónica no mostró diferencias espaciales significativas $(\mathrm{KW}=2.21, p>0.05$; Fig. $4 \mathrm{D})$, a pesar que en el grupo de las estaciones internas se contabilizó mayor abundancia (W=187, $p>0.05$; Fig. 4E). La distribución de la abundancia zooplanctónica con respecto a la ubicación de las estaciones fue revelada por la ordenación MDS (Stress= 0.16); no se evidenció una clara separación entre las dos zonas en que se dividió el estudio, sin embargo, se observa más asociación entre las estaciones externas (Fig. 4F). 
El ANOSIM entre la abundancia zooplanctónica y los períodos de estudio (surgencia y relajación) mostró un $R$ global de 0.35 y un nivel de significancia de $0.1 \%$ (interpretado como un valor de $p=0.001$ ), demostrando diferencias significativas entre ambos períodos. No obstante, el análisis de ANOSIM aplicado entre la abundancia y las zonas de estudio (externas e internas), no arrojó diferencias significativas ( $R$ global de 0.053 ).

El SIMPER, indicó que la disimilitud entre la estructura comunitaria entre los dos períodos fue del 63.7\% (Tabla 2), el mayor aporte en surgencia correspondió solamente a los copepoditos, mientras que en relajación, la contribución estuvo repartida entre las larvas de crustáceos, Oithona nana, Paracalanus quasimodo y Acartia tonsa.

El ACC en surgencia mostró la varianza explicada que vincula el componente 1 con el componente 2 fue del 53\%. El componente 1, alcanzó una correlación con el conjunto de especies de 0.91 , se correlacionó Nanocalanus minor con el oxígeno disuelto y temperatura, inverso con Oithona nana, nauplios de crustáceos y con los nitratos. En el componente 2, la correlación con el conjunto de especies fue de 0.83 , donde se encuentra correlacionada directamente Oikopleura dioica con salinidad y nitrato en forma inversa las larvas de peces se correlacionan de forma directa con la temperatura (Fig. 6A).

El ACC en relajación obtuvo una varianza explicada que vincula el componente 1 con el componente 2 del $40 \%$. Además el primer eje, obtuvo una correlación con el conjunto de especies de 0.91 , se caracterizó por un lado, con los huevos de peces, copepoditos y $A$. tonsa y en relación inversa con la temperatura y nitrato. Mientras que el componente 2 , que tuvo una correlación con el conjunto de especies de 0.87 , estuvo mayormente caracterizado por el nitrito y fosfato en relación inversa con la salinidad. La especie más afín a estos elementos fue $P$. quasimodo (Fig. 6B).

\section{Discusión}

La variabilidad temporal de la temperatura en la laguna El Morro presentó el comportamiento típico del fenómeno de surgencia costera en las lagunas costeras del Nororiente del país (Palazón 2000) y de la isla de Margarita (Palazón et al. 1996, Palazón \& Penoth 1998). Según Palazón y Penoth (1998), los patrones temporales observados en los cuerpos de agua costeros están directamente relacionados con los cambios de la temperatura atmosférica y la dinámica del mar adyacente.

La temperatura media $\left(29.7^{\circ} \mathrm{C}\right)$, fue superior a la reportada en estudios realizados en otras lagunas costeras de la isla de Margarita como Laguna de Boca de Palo $\left(27.7^{\circ} \mathrm{C}\right)$ por Palazón y Penoth (1998); Laguna de Las Marites $\left(28.14^{\circ} \mathrm{C}\right)$ por Palazón et al. (1996); Laguna de Punta de Piedras $\left(28.5^{\circ} \mathrm{C}\right)$ por Pereira (2006); Laguna de Los Mártires $\left(28.12{ }^{\circ} \mathrm{C}\right)$ reportado por Fontanive et al. (2010). Posiblemente la alta temperatura en la laguna El Morro se deba a lo somero del cuerpo de agua, además de un efecto de las mareas disminuido (Palazón 2000).

Con respecto a la variación estacional de la temperatura, está se caracterizó por la presencia de zonas cálidas principalmente hacia el interior de la laguna, lo cual podría deberse a la baja profundidad, alta incidencia solar, elevada evaporación, además de la absorción de calor por parte de la materia en suspensión (Palazón 2000).
La salinidad media (35 ups) registrada durante los meses de estudio en la laguna costera El Morro, difiere de la condición hipersalina comúnmente observada en las lagunas costeras del Oriente del país y de la Isla de Margarita (Cervigón \& Gómez 1986), por lo que se puede pensar que existe ingreso constante de agua marina al espejo de agua de manera frecuente a través de la boca que comunica a la laguna con la zona costera de Guaraguao, además puede estar ocurriendo infiltración de agua de mar de las playas aledańas, o por el contrario, existen otros factores ambientales o antropogénicos (escorrentías o descargas de aguas de origen doméstico), que causan una disolución de las concentraciones de sales. El valor promedio obtenido fue inferior al reportado por Pereira (2006) para la Laguna de Punta de Piedras (38.34 ups); en cambio fue superior al valor obtenido (8.88 ups) por Fontanive et al. (2010) para la Laguna de Los Mártires, que tiene como característica el aporte de un riachuelo.

$\mathrm{Al}$ igual que la temperatura, los valores más altos de salinidad se registraron en las estaciones del interior del cuerpo lagunar, debido al poco intercambio de agua con la playa adyacente, la elevada evaporación y la poca profundidad de la columna de agua. Este contraste de la salinidad superficial, concuerda con lo reportado en lagunas de la zona sur de la Isla de Margarita (Palazón et al. 1996).

Los registros de oxígeno disuelto detectados en el presente estudio fueron superiores a los mencionados por Cervigón y Gómez (1986), los cuales afirman que las lagunas costeras de la isla de Margarita suelen presentar concentraciones de oxígeno disuelto entre 2,93 y $3,48 \mathrm{mg} . \mathrm{L}^{-1}$ y rara vez superan los $6 \mathrm{mg} . \mathrm{L}^{-1}$, debido al carácter reductor de los sedimentos y la alta demanda química y biológica. Los valores elevados de oxígeno disuelto sugieren el aporte de los procesos involucrados en la oxigenación de la columna de agua como la actividad fotosintética (aunque no fue medido en este estudio), procesos de mezcla por efecto de los vientos y las mareas (Fontanive et al. 2010).

La concentración media de nitrato $\left(0.12 \mathrm{mg} . \mathrm{L}^{-1}\right)$ fue inferior a lo reportado por Palazón et al. (1996) para la Laguna de Las Marites (0.0843 mg. $\left.\mathrm{L}^{-1}\right)$ y Fontanive et al. (2010) para la Laguna de Los Mártires (0.122 mg. $\left.\mathrm{L}^{-1}\right)$, en cambio, fue superior a los obtenidos por Palazón y Penoth (1998) para la Laguna de Boca de Palo (0.01 mg. $\left.\mathrm{L}^{-1}\right)$. El nitrato es el compuesto nitrogenado más estable y, por lo tanto, suele ser abundante en los cuerpos de agua (Palazón et al. 1996, Carpenter \& Capone 2016). Según Duarte et al. (2017) la degradación u oxidación del nitrógeno inorgánico en el ambiente se realiza gracias a la intervención bacteriana, en donde el nitrato es el resultado del metabolismo de dichos organismos, y por lo tanto, es la especie inorgánica nitrogenada menos tóxica presente en los cuerpos de agua.

La concentración media de nitrito $\left(0.04 \mathrm{mg} . \mathrm{L}^{-1}\right)$ fue inferior a la de los otros compuestos nitrogenados; asimismo, los valores también fueron inferiores a los reportados para otras lagunas costeras de Nueva Esparta (Palazón et al. 1996, Palazón 2000, Fontanive et al. 2010), debido a que esta especie química es un elemento transitorio en el ciclo del nitrógeno, y por lo tanto, es rápidamente oxidado a nitrato en condiciones aeróbicas (Carpenter \& Capone 2016).

A pesar que las concentraciones de fosfato fueron relativamente bajas (media: 0.03 mg. $\mathrm{L}^{-1}$ ), siguen siendo superiores a los reportados por Palazón et al. (1996) en la Laguna de Las 
Tabla 2. Porcentaje de contribución de los principales grupos del zooplancton que marcaron la disimilitud entre los dos períodos estudiados en el análisis SIMPER. P. Abun surgencia: abundancia promedio en surgencia, P. Abun Relajación: abundancia promedio relajación, P. Diss: promedio de disimilaridad. Porcentaje de disimilaridad: $63.7 \%$

\begin{tabular}{|c|c|c|c|c|c|}
\hline \multirow[t]{2}{*}{ Especies } & \multirow{2}{*}{$\begin{array}{l}\text { P. Abun. } \\
\text { Surgencia }\end{array}$} & \multirow{2}{*}{$\begin{array}{l}\text { P. Abun. } \\
\text { Relajación }\end{array}$} & \multirow[t]{2}{*}{ P.Diss } & \multicolumn{2}{|c|}{ Porcentaje } \\
\hline & & & & Contribución & Acumulado \\
\hline Larvas de crustáceos & 24.61 & 80.95 & 1.41 & 20.83 & 20.83 \\
\hline Oithona nana & 6.78 & 66.66 & 1.24 & 18.16 & 38.99 \\
\hline Paracalanus quasimodo & 2.26 & 29.40 & 0.91 & 8.36 & 47.34 \\
\hline Copepoditos & 26.01 & 15.64 & 1.12 & 7.41 & 54.75 \\
\hline Nanocalanus minor & 11.67 & 18.43 & 1.14 & 6.57 & 61.33 \\
\hline Paracalanus aculeatus & 1.50 & 18.39 & 1.41 & 5.67 & 66.99 \\
\hline Acartia tonsa & 20.22 & 23.71 & 1.47 & 5.58 & 72.58 \\
\hline Euterpina acutifrons & 4.56 & 14.69 & 1.28 & 4.37 & 76.95 \\
\hline Oithona sp. & 9.06 & 7.46 & 1.11 & 3.98 & 80.96 \\
\hline
\end{tabular}

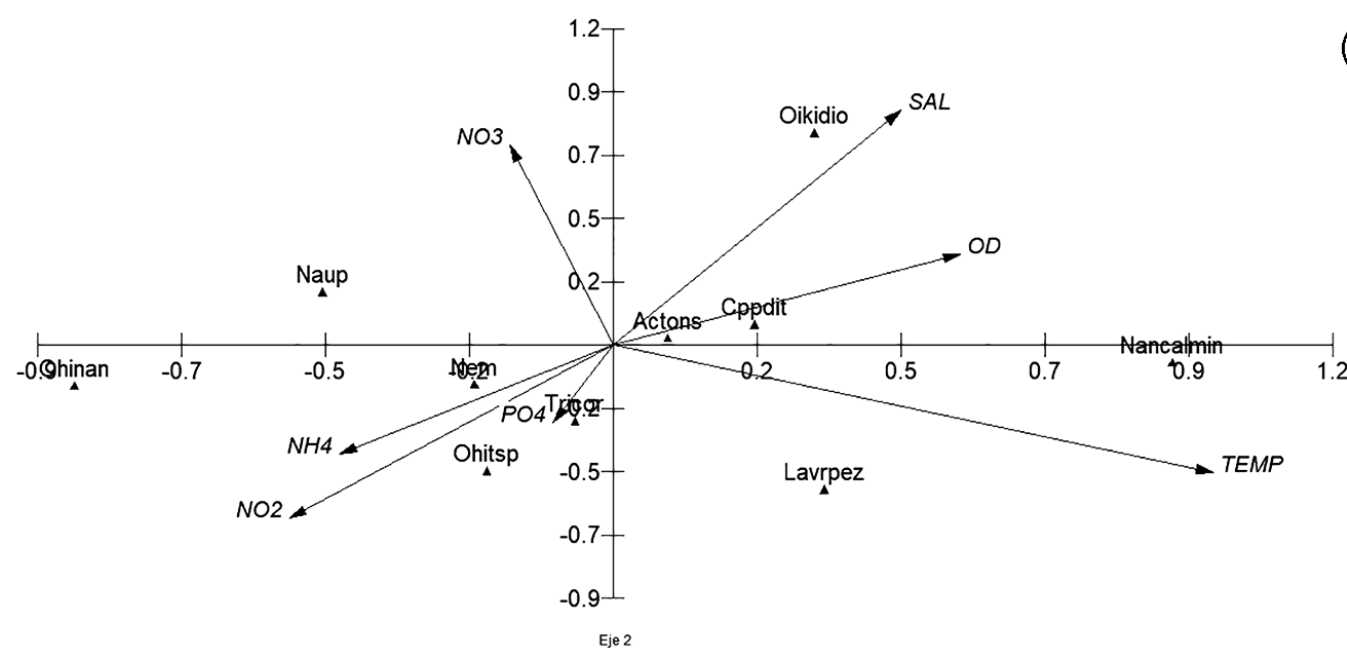

(A)

Vector scalina: 1.30

Eje 2

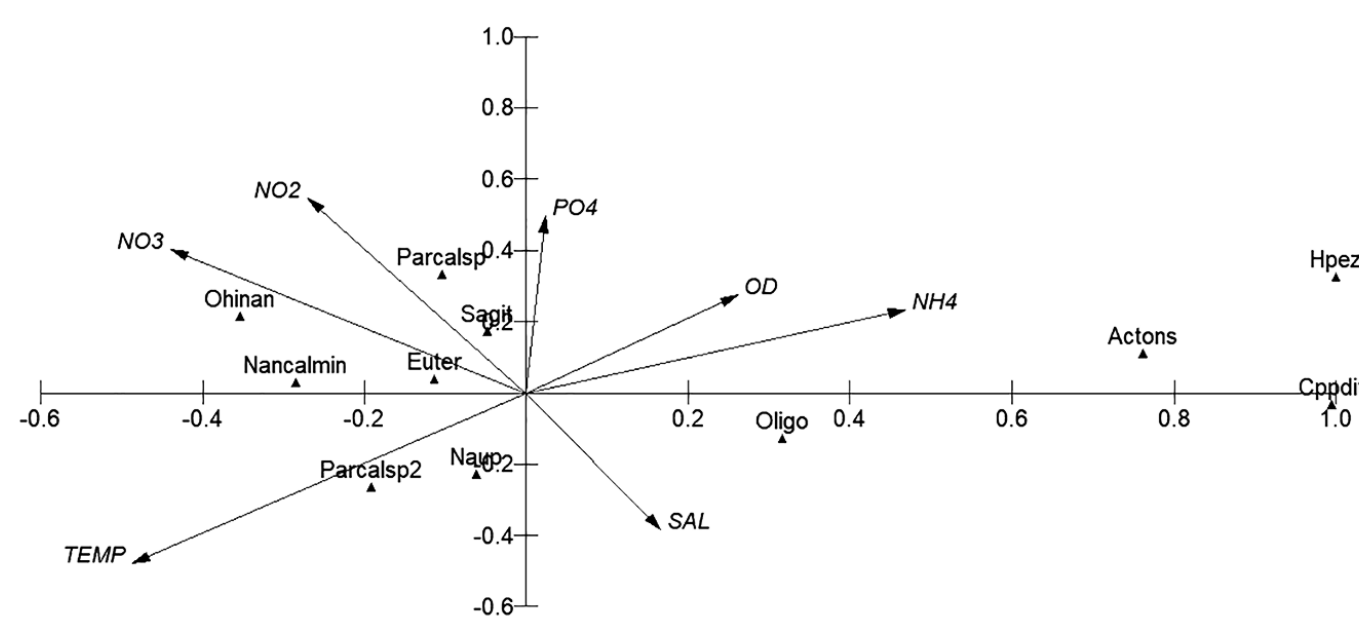

(B)

Vector scalina: 1.10

Eje 2

Figura 6.- Proyección ortogonal del ACC en surgencia (A) y relajación (B) entre las especies de zooplancton que aparecieron en porcentaje mayor al $10 \%$ y las variables ambientales medidas en la Laguna del Morro, Isla de Margarita, Venezuela. SAL: salinidad, TEMP: temperatura, OD: oxígeno disuelto, $\mathrm{NO}_{2}$ : nitrito, $\mathrm{NH}_{4}$ : $\mathrm{Amonio}_{\mathrm{NO}} \mathrm{NO}_{3}$ : nitrato, Oikidio: Oikopleura dioica, Naup: nauplio de custáceos, Actons: Acartia tonsa, Cppdit: Copepoditos, Ohina: Oithona nana, Nem: nematodos, Ohitsp: Ohitona sp., Lavrpez: larvas de peces, Nancalmin: Nanocalanus minor. Paracal: Paracalanus quasimodo, Euter: Euterpina acutifrons. 
Marites (0.088 mg.L $\left.\mathrm{L}^{-1}\right)$, Palazón (2000) para la Laguna del Saco (0.0230 mg. $\left.\mathrm{L}^{-1}\right)$. En general, en las lagunas costeras las concentraciones de fosfato pueden incrementarse por la ocurrencia de procesos de resuspensión y remineralización como resultado de la acción del viento sobre el cuerpo de agua poco profundo (López-Hernández et al. 1985, Kennish et al. 2014), y también por la descomposición de la materia orgánica de origen vegetal como los manglares (Nielsen \& Anderson 2003). Sin embargo, los compuestos y residuos de origen antropogénico son la mayor fuente de fosfato en los medios acuáticos, como por ejemplo, los detergentes, fertilizantes, y aguas residuales (Rodrigues et al. 2016). La persistencia y difícil remoción del fosfato lo caracteriza una de las principales causas de eutrofización en las aguas salobres y dulces (Fontanive et al. 2010).

La variabilidad de la abundancia del zooplancton mostró una respuesta temporal marcada, con los registros más altos durante el período de relajación. Esta tendencia es contradictoria cuando se compara con otros trabajos realizados en la Isla de Margarita y zonas adyacentes (Gómez \& Hernández 2008, Hernández-Ávila \& Gómez-Gaspar 2014, GómezGaspar 2016). Típicamente se ha considerado al proceso de surgencia costera como el principal modulador del sistema marino-costero del nororiente de Venezuela (Müller-Karger et al. 2004, Alvera-Azcárate et al. 2011). Sin embargo, hay que tomar en cuenta que esta laguna tiene una comunicación limitada con el mar, únicamente por la boca, por lo que posiblemente el efecto de la surgencia costera no llega hasta la zona interna de la laguna, favoreciendo el efecto de escorrentía, resuspensión de los sedimentos, por la poca profundidad, además de posibles indicios de enriquecimiento del agua por nutrientes, demostrados en el ACC en la época de relajación que coinciden con la temporada de lluvias en la Isla (LópezMonroy \& Troccoli-Ghinaglia 2015), debido a la correlación ente el nitrito y fosfato, evidenciando posiblemente los aportes de aguas residuales urbanas, así como el estiércol de las aves. Otra posible factor, que pudo influir en la abundancia mayor en relajación, pudo ser debida al ecosistema de manglar que rodea a la laguna, el cual sirve como zonas de desove, crianza y hábitat de macroinvertebrados móviles (como cangrejos y camarones) y sésiles (como moluscos, cirrípedos, colonias hidroides). Muchas especies marinas de importancia pesquera son parcial o totalmente estuarinas dependientes y de acuerdo a sus estrategias alimenticias y reproductivas emplean las lagunas costeras como áreas de reproducción, alimentación, crianza y protección (Yáñez-Arancibia et al. 2014).

Debe considerarse que aunque la laguna El Morro este relativamente aislada de la porción marina circundante, debido a la estrecha boca, puede presentar influencia del ambiente marino en mayor o menor grado. Kaiser et al. (2005) mencionan que este tipo de cuerpos de agua son lugares altamente favorables para diferentes especies de organismos marinos. Esto último quedo corroborado con la mayor diversidad y abundancia de especies marinas (Tabla 1 ).

Se identificaron 15 grupos de organismos zooplanctónicos en la laguna El Morro, un número semejante al reportado por Hernández (2000) y Marcano (2003) para otras lagunas costeras de la isla de Margarita y del centro-este del país (Zoppi 1974). No obstante, difiere de Gómez (1983) quien reportó mayor cantidad de grupos (22) en la Laguna de la Restinga, isla de Margarita. Al comparar la composición con los trabajos mencionados anteriormente los copépodos fueron el grupo dominante $(46.7-85.7 \%)$, seguido de larvas de crustáceos y apendiculados. Sin embargo, en la presente investigación las larvas de crustáceos fueron las más abundantes. Los resultados de esta investigación son muy similares a los encontrados por Zoppi (1974) en la laguna de Tacarigua, indicando que dicha composición se caracteriza por la pobreza en variedad y porque las comunidades están formadas por una alta densidad de pocas especies permanentes y dominantes, típicas de aguas salobres y que definen bien a las lagunas costeras del centro-este del país.

Al comparar la abundancia de la laguna El Morro, con otras lagunas de la Isla de Margarita, resulta alta, pues su media fue de 110685 ind. $\mathrm{m}^{-3}$, mientras que en la entrada de la laguna de la Restinga, Gómez (1983) reportó 10040 org. $\mathrm{m}^{-3}$, y en la laguna de las Marites, Hernández (2000) halló abundancias entre 2166 - 188083 org. $\mathrm{m}^{-3}$. No obstante, resultados similares fueron encontrados en otras lagunas costeras del país, por Zoppi (1974), lagunas de Tacarigua y Unare (128362 y 191914 org. m ${ }^{-3}$, respectivamente).

Los pocos grupos zooplanctónicos registrados en este estudio, coincide con la opinión de que en las lagunas costeras se encuentra generalmente una menor diversidad de especies que en zonas costeras aledańas con menor variabilidad ambiental (Hemraj et al. 2017). Se sabe que en las lagunas costeras el componente holoplanctónico tiende a ser menos diverso que el meroplanctónico y además que hay una relación menos independiente entre la fauna planctónica y bentónica (Hemraj et al. 2017). En las aguas someras de la laguna El Morro, la relación entre el plancton y el bentos es muy estrecha; en la capa superficial del agua se encontraron grupos bentónicos como los oligoquetos y turbelarios (Tabla 1), los cuales ascienden a la columna de agua por movimientos migratorios verticales por transporte de agua del fondo hacia la superficie, por efecto de la turbulencia del agua y/o por remoción del fondo por la acción del instrumento de muestreo.

En la laguna El Morro, los grupos de zooplancton más abundantes en orden descendente y que a su vez marcaron la diferencia entre los dos períodos estudiados son: las larvas de decápodos, los copépodos, los huevos y larvas de peces y los quetognatos. En contraste, con los estudios de zooplancton en zonas cercanas (Gómez \& Hernández 2008, Hernández-Ávila \& Gómez-Gaspar 2014), los copépodos son el grupo predominante, al que le siguen los cladóceros, los apendicularios, los quetognatos y las larvas de crustáceos. Esta diferencia puede atribuirse a la mayor influencia oceánica que recibe la costa sureste de margarita, lo que permite una mayor composición de organismos holoplanctónicos.

Como se mencionó anteriormente, las larvas de crustáceos representaron el grupo más importante de la comunidad zooplanctónica en la laguna El Morro, cuya abundancia varió entre los diferentes meses analizados. En julio y agosto se registraron las mayores abundancias, principalmente de nauplios, zoeas y larvas de cirrípedos. El hecho de haber encontrado en esta laguna a las larvas de decápodos con gran abundancia durante casi todos los muestreos puede estar asociado a una actividad reproductiva durante todo el año. Otros factores que promueven la mayor abundancia de las larvas de decápodos sobre los demás grupos en la laguna es la estrecha relación de éstas con 
poblaciones de adultos de aguas someras y la advección a que ellas estarían sometidas por efecto de las corrientes de marea y otros procesos de mezcla vertical (Mujica \& Navas 2010). Esto no fue corroborado con el MDS; sin embargo, las abundancias más altas de las larvas de crustáceos decápodos se contabilizaron en las estaciones internas.

Como se mencionó anteriormente, la abundancia alta de larvas de crustáceos decápodos se debió a la presencia de los adultos, ya que Maza (1986) reportó 29 especies de crustáceos del orden decápoda en esta laguna, siendo las especies más abundantes: Callinectes ornatus, Penaeus sp. y Petrolisthes armatus. Incluso la primera especie es explotada para la subsistencia de las poblaciones aledańas de la laguna, de igual manera ocurre con el gasterópodo Melongena melongena. Por lo tanto, esta laguna posiblemente sea importante para la producción de las poblaciones de crustáceos, considerándolo un reservorio natural, además preserva este grupo que también es de importancia comercial (Pfaff et al. 2015).

Los copépodos constituyeron el segundo grupo en importancia, su riqueza y abundancia también varió temporalmente. Durante el período de surgencia dominaron las especies $A$. tonsa y $N$. minor. En el período de relajación se encontró una mayor riqueza de especies, con una dominancia de $O$. nana, seguida por P. quasimodo, A. tonsa y N. minor y dentro de los Harpacticoides se registró a $E$. acutifrons.

La presencia de $A$. tonsa, $P$. quasimodo y $N$. minor como especies abundantes y dominantes en este estudio coincide con los antecedentes sobre la fauna de copépodos dominantes en la zona NE de Venezuela, aguas de Margarita (Laguna de la Restinga), Fosa de Cariaco y Golfo de Cariaco (Legaré 1961, Zoppi 1961, Márquez et al. 2006, 2011), así como del Mar Caribe y Caribe suroriental (Michael \& Foyo 1976, Razouls et al. 2005) y sur del Golfo de México (Campos-Hernández \& Suárez-Morales 1994, Suárez-Morales et al. 1996). Por su parte, $A$. tonsa ha sido reportada como una especie que presenta un amplio ámbito de tolerancia de salinidad y temperatura, por lo que ha sido registrada con gran éxito en medios estuarinos, en lagunas costeras con fuerte influencia marina y en zonas con influencia terrígena (Peck et al. 2015). Todo lo antes expuesto, coincide con los resultados obtenidos en la presente investigación y corroborado con el ACC, ya que $A$. tonsa se registró durante los dos períodos estudiados y correlacionada en la época de relajación con la salinidad. El Cyclopoida O. nana, se distribuyó en todo el sistema lagunar y durante todos los meses del estudio; es un copépodo común en aguas tropicales, subtropicales y templadas, eurihalino, epipelágico, frecuente en los sistemas lagunares y estuarinos de Venezuela, Colombia y México (Zoppi 1974, Hernández 2000, Álvarez-Silva et al. 2006, Álvarez-Silva \& Torres-Alvarado 2013, López-Peralta \& Mojica-López 2015). Sin embargo, resultados contrarios fueron reportados por Álvarez-Silva et al. (2006) y Álvarez-Silva y Torres-Alvarado (2013) para sistemas lagunares de México, quienes indicaron a $O$. nana como habitantes temporales ya que solo estuvieron presentes algunos meses.

Un tercer grupo estuvo conformado por los huevos y larvas de peces, principalmente de Engraulidos y Clupeidos. Gómez y Hernández (2008) mencionan que las costas este y sureste de Margarita son las áreas predominantes de desove de la sardina, Sardinella aurita. Por lo tanto, no es raro haber encontrado hue- vos y larvas en esta zona, ya que la laguna EL Morro se encuentra ubicada en la costa sur-este y por efectos de corrientes pudieron entrar en ella. Por otra parte, se encontraron huevos todos los meses de estudio, con las abundancias máximas en abril y agosto, lo que indica un relativo buen desove durante esos meses, pero inusual porque el desove máximo de la sardina se presenta de noviembre a marzo (Freón et al. 1997, Gassman et al. 2014).

Es importante destacar la presencia de individuos expatriados de especies marinas, principalmente durante el período de surgencia, y que incidentalmente pudieron penetrar a la laguna, sin tener actividad reproductiva en ella y por tanto su distribución se encontró restringida a las estaciones de la zona externa. Tales especies pudieron ingresar al sistema lagunar entre los meses de marzo a mayo, cuando la boca de la laguna permite el intercambio con el medio marino adyacente, permitiendo la existencia de organismos marinos en el interior de la laguna, entre los que se encuentra el cladócero $P$. avirostris, el decápodo B. faxoni, el tunicado $O$. dioica y varias especies de eufásidos, ostrácodos y quetognatos, y dentro del meroplancton se registraron a las larvas de cirrípedos, equinodermos y moluscos.

También debe considerarse importante la presencia de dos especies, típicas de agua dulce, principalmente en la época de relajación, como son el rotífero Brachionus plicatilis y el cladócero Moina micrura. Los rotíferos normalmente presentan la mayor riqueza de especies en agua dulce (Matsumura-Tundisi et al. 1990), además representan el grupo más extendido en los embalses y sistemas lagunares de Venezuela (Zoppi 1974, Briceño et al. 2009) por tener estrategias reproductiva del tipo selección $r$, lo cual los hace animales oportunistas y con una tolerancia alta a las fluctuaciones en las condiciones ambientales (Reverol et al. 2008, Astiz \& Álvarez 2014) lo que les permite una distribución amplia (Sendacz 1993), y la posibilidad de colonizar rápidamente ambientes perturbados.

El escenario general para la Laguna El Morro, involucra la estacionalidad (surgencia-relajación) como un efecto importante en la distribución y abundancia del zooplancton. Dentro de esta, existen grupos que presentan variaciones importantes en su distribución espacial, con capacidad de dispersión en zonas particulares, según su plasticidad adaptativa a los cambios ambientales. Es posible que las escalas espaciales y temporales de muestreo hayan condicionado el patrón de distribución de los grupos identificados. Al respecto, Albaina e Irigoien (2007) señalan que las limitaciones en la comprensión y predicción de las distribuciones de plancton en regiones altamente dinámicas surgen de un desfase entre las escalas en las cuales los datos biológicos y físicos son obtenidos, y las escalas en las que las estructuras de mesoescala influencian a estas comunidades. Por lo anterior se concluye que este sistema debe ser monitoreado con mayores esfuerzos de muestreo, para conocer con mayor detalle la dinámica de la comunidad zooplanctónica que en él se desarrolla.

\section{Agradecimientos}

El primer autor quiere agradecer al Centro Regional de Investigaciones Ambientales (CRIA - UDONE) por la facilidad de los equipos y todo el apoyo logístico. Este trabajo fue realizado con el financiamiento del proyecto Gestión ambiental para el saneamiento de la laguna costera El Morro, municipio Mariño, isla de Margarita (Proyecto LOCTI, Código PL-016). 


\section{Literatura citada}

Albaina A. \& X. Irigoien. 2007. Fine scale zooplankton distribution in the Bay of Biscay in spring 2004. Journal of Plankton Research 17(10): 851-870. https://doi.org/10.1093/plankt/ $\mathrm{fbm} 064$

Álvarez-Silva C., G. Miranda-Arce, G. De Lara-Issasi \& S. GómezAguirre. 2006. Zooplancton de los sistemas estuarinos de Chantuto y Panzacola, Chiapas, en época de secas y lluvias. Hidrobiológica 16(2): 175-182.

Álvarez-Silva C. \& M.R. Torres-Alvarado. 2013. Composición y abundancia del zooplancton de la laguna de Coyuca, Guerrero, México. Hidrobiológica 23(2): 241-249.

Alvera-Azcárate A., A. Barth, R.H. Weisberg, J.J. Castañeda, L. Vandenbulcke \& J.M. Beckers. 2011. Thermocline characterization in the Cariaco basin: A modelling study of the thermocline annual variation and its relation with winds and chlorophyll-a concentration. Continental Shelf Research 31 (1): 73-84. https://doi.org/10.1016/j.csr.2010.11.006

American Public Health Association, American Water Works Association, Water Environment Federation (APHA-AWWAWEF). 1998. Standards Methods for the examination of water and wastewater. 20th Edition, American Public Health Association, Washington DC. 950 pp.

Aranguren-Riaño N., C. Guisande \& R. Osuna. 2011. Factors controlling crustacean zooplankton species richness in Neotropical lakes. Journal of Plankton Research 33(8): 1295-1303. https://doi.org/10.1093/plankt/fbr028

Astiz S. \& H. Álvarez. 2014. Dinámica del zooplancton y su relación con la calidad de agua en el río Cataniapo, Amazonas, Venezuela. Ecotrópicos 27(1-2):13-30 2014

Bevilacqua M., L. Cárdenas \& D. Medina. 2006. Las áreas protegidas en Venezuela: diagnóstico de su condición 1993/2004. Fundación Empresa Polar Acoana VICN. Caracas, Venezuela. $120-122 \mathrm{pp}$.

Boadas M. 1985. Taxonomía, distribución y abundancia de los crustáceos de la laguna El Morro de Porlamar, isla de Margarita. Tesis, Licenciado en Biología. Escuela de Ciencias, Universidad de Oriente, Cumaná, Venezuela.91pp.

Boltovskoy D. 1999. South Atlantic zooplankton. Vol. I y II. Leiden: Backhuys Publishers.

Boyer J., J. Fourqrean \& R. Jones. 1997. Spatial characterization of water quality in Florida Bay and Whitewater Bay by multivariate analyses: Zones of similar influence. Estuaries 20(4):743-758. https://doi.org/10.2307/1352248

Briceño H., R. Buonocore, C. Sangronis, L. García-Pinto, J. Rojas, J. Chirinos, A. González \& C. López. 2009. Composición y abundancia del plancton de la Costa Noreste de la Bahía El Tablazo, Sistema de Maracaibo, Venezuela. Boletín del Centro de Investigaciones Biológicas 43(4): 463-485.

Campos-Hernández A. \& E. Suárez-Morales. 1994. Copépodos pelágicos del Golfo de México y Mar Caribe. 1. Biología y Sistemática. Centro de Investigaciones de Quintana Roo. (CIQROII CONACYT. México.

Carpenter E.J. \& D.G. Capone. 2016. Nitrogen in the marine environment. 2nd Edition. Elsevier. 1757 pp.

Clarke K. \& R. Gorley. 2006. PRIMER v5 (and v6): User manual/ tutorial. Plymouth: PRIMER-E.

Cervigón F. \& A. Gómez. 1986. Las lagunas litorales de La Isla de Margarita. Fundación Científica Los Roques. Caracas, Venezuela. $89 \mathrm{pp}$.

Duarte I.D., N.H. Silva, I. da Costa Souza, L. de Oliveira, L.D. Rocha, M. Morozesk \& S.T. Matsumoto. 2017. Water quality of a coastal lagoon (ES, Brazil): abiotic aspects, cytogenetic damage, and phytoplankton dynamics. Environmental Science and Pollution Research 24 (11): 10855-10868. https://doi.org/10.1007/s11356-017-8721-2.

Esnal G.B. 1999. Appendicularia. In: D. Boltovskoy, eds. Zooplankton of the South Atlantic Ocean. Leiden: Backhuys. Pp. 1375-1399.

Ferrol-Schulte D., M. Wolff, S. Ferse \& M. Glaser. 2013. Sustainable Livelihoods Approach in tropical coastal and marine socialecological systems: A review. Marine Policy. 42: 253-258. https://doi.org/10.1016/j.marpol.2013.03.007.
Fontanive P., J.L. Fuentes, J.C. Salazar, J. López \& P. López. 2010. Condición trófica de la Laguna de Los Mártires, Isla de Margarita, Venezuela, periodo enero-diciembre 2006. Ciencia 18(2): $81-90$.

Freón P., M. El Khattabi, J. Mendoza \& R. Guzmán. 1997. Unexpected reproductive strategy of Sardinella aurita off the coast of Venezuela. Marine Biology 128(3): 363-372. https://doi. org/10.1007/s002270050102

Gassman J., N. Eslava, L. González \& N. Aguado. 2014. Indicadores reproductivos de la sardina Sardinella aurita (Clupeiformes: Clupeidae) del sureste de la isla de margarita, Venezuela. Boletín del Centro de Investigaciones Biológicas 46(2): 121-136.

Gómez A. 1983. Pigmentos clorofílicos, producción primaria y abundancia planctónica en el canal de entrada a la laguna de La Restinga, Venezuela. Boletín del Instituto Oceanográfico de Venezuela 22 (1 y 2):43-63.

Gómez A. \& I. Hernández. 2008. Abundancia interanual del zooplancton nocturno en la costa este de Isla Margarita, Venezuela. Boletín del Instituto Oceanográfico de Venezuela 47(2): 91-102.

Gómez-Gaspar A. 2016. Densidad de huevos de sardina Sardinella aurita, abundancia de zooplancton e hidrografía en la península de Araya y sur de Isla Margarita, Venezuela. Boletín de Investigaciones Marinas y Costeras 45 (1): 57-72.

Gutiérrez M.E., E. Suarez-Morales, M. Gutiérrez-Aguirre, M.S. Briano, J. Granados-Ramírez \& T. Garfias-Espejo. 2008. Cladócera y Copepoda de las aguas continentales de México. Guía ilustrada. Universidad Autónoma de México y El Colegio de la Frontera Sur. México. 322pp.

Hemraj D.A., M.A. Hossain, Q. Ye, J.G. Qin \& S.C. Leterme. 2017. Plankton bioindicators of environmental conditions in coastal lagoons. Estuarine, Coastal and Shelf Science 184: 102-114. https://doi.org/10.1016/j.ecss.2016.10.045

Hernández G. 2000. Zooplancton superficial de la Laguna de Las Marites, isla de Margarita, Venezuela en el periodo junionoviembre 1999. Tesis, Licenciado en Biología, Escuela de Ciencias Aplicadas del Mar, Universidad de Oriente, Venezuela. 145 pp.

Hernández-Ávila I. \& A. Gómez-Gaspar. 2014. Patrones de abundancia y composición del zooplancton costero a varias escalas temporales en un ciclo de surgencia estacional en la Isla de Margarita, Venezuela. Interciencia 39(2): 122-128.

Hernández-Trujillo S., G. Esqueda-Escárcega \& R. Palomares-García. 2010. Variabilidad de la abundancia de zooplancton en Bahía Magdalena Baja California Sur, México (1997-2001). Latin American Journal of Aquatic Research 38(3):438-446. DOI: 10.3856/vol38-issue3-fulltext-8.

Kaiser M.J., M.A. Atrill, S. Jennings, D. Thomas, D.K. Barnes, A.S. Brierley, N. Polunin, D. Rafaelli \& P.E. Williams. 2005. Marine Ecology Process, Systems and Impacts. Oxford University Press, Oxford, 557 pp

Kennish M.J., M.J. Brush \& K.A. Moore. 2014. Drivers of change in shallow coastal photic systems: an introduction to a special issue. Estuaries and coasts 37(1): 3-19. https://doi. org/10.1007/s12237-014-9779-4

Lankford R. R. 1977. Coastal Lagoons of Mexico, their origin and classification, in L.E. Cornin (Ed.), Estuarine Processes, Circulation, Sediments and Transfer of Material in the Estuary, Academic Press, New York, p. 182.

Legaré J.E. 1961. Estudios preliminares del zooplancton en la región de Cariaco. Boletín Instituto Oceanográfico 1(1): 191-218.

Legendre P \& L. Legendre. 1998. Numerical Ecology. 2da. Edition. Elsevier Sciencie, Netherlands.

Longhurst A. \& D. Pauly. 1987. Ecology of Tropical Oceans. San Diego, EE.UU.: Academic Press, 407 pp.

López-Hernández D., J. Rodríguez \& N. Duarte. 1985. Parámetros que controlan los niveles de fósforo en aguas y sedimentos en una laguna costera (Laguna de Tacarigua). Boletín del Instituto Oceanográfico de Venezuela 24(1-2): 225-236.

López I. 2012. Caracterización físico-química y microbiológica de la laguna El Morro, Municipio Mariño, isla de Margarita, durante el periodo marzo 2011-marzo2012. Tesis, Licenciado en Biología Marina, Escuela de Ciencias Aplicadas del Mar, Universidad de Oriente, Venezuela.75 pp. 
López-Monroy F. \& L. Troccoli-Ghinaglia. 2015. Aproximación sobre la climatología de la isla de Margarita y su importancia en los procesos oceánicos. Saber 26(4): 467-471

López-Peralta, R. H. \& L. H. Mojica López. 2015. Especies de Oithona (Crustacea: Copepoda) en el pacífico colombiano en el segundo periodo lluvioso de 2001. Revista Facultad de Ciencias Básicas Universidad Militar Nueva Granada, 11 (2): 38-53. DOI http://dx.doi.org/10.18359/rfcb.1298

Lorenzoni L., G.T. Taylor, C. Benitez-Nelson, D.A. Hansell, E. Montes, R. Masserini, K. Fanning, R. Varela, Y. Astor, L. Guzmán \& F.E. Muller-Karger. 2013. Spatial and seasonal variability of dissolved organic matter in the Cariaco Basin. Journal of Geophysical Research: Biogeosciences. 118: 951-962. http://dx.doi.org/10.1002/jgrg.20075.

Marcano A. 2003. Composición y abundancia del zooplancton del eje Pampatar (Punta Ballena)- La Isleta, isla de Margarita, Venezuela en el periodo febrero-julio 2002. Tesis, Licenciado en Biología Marina, Escuela de Ciencias Aplicadas del Mar, Universidad de Oriente, Venezuela.62 pp.

Márquez B., B. Marín., E. Zoppi \& C. Moreno. 2006. Zooplancton del Golfo de Cariaco. Boletín del Instituto Oceanográfico de Venezuela 45(1): $61-78$.

Márquez B., L. Troccoli, L.M. Marcano, J. Morales, T. Allen, B. Marín \& R. Díaz-Ramos. 2011. Estructura comunitaria del zooplancton en dos localidades del golfo de cariaco, Venezuela. Boletín del Instituto Oceanográfico de Venezuela. 50 (2): 103-119

Matsumura-Tundisi T., S.N. Leitão, L.S. Aguena \& J. Miyahara. 1990. Eutrofização da represa de Barra Bonita: Estrutura e organização da comunidade de Rotifera. Revista Brasileira de Biologia 50: 923-935

Maza M. 1986. Taxonomía, distribución y abundancia de las especies de peces de la laguna del Morro de Porlamar, isla de Margarita, estado Nueva Esparta. Tesis. Licenciado en Biología Marina, Escuela de Ciencias Aplicadas del Mar, Universidad de Oriente, Venezuela. 94 pp.

Michael H.B. \& M. Foyo. 1976. Caribbean Zooplankton. Part I. Shiphonophora, Heteropoda, Copepoda, Euphausiacea, Chaetognatha and Salpidae. Office of Naval Research Department of Navy. Bethesda, Maryland. 712 p.

Mujica, A. 2007. Dispersión de larvas de crustáceos decápodos en canales y fiordos de la Región de Aysén, Chile. Revista de Biología Marina y Oceanografía. 42(3): 231-240. http:// dx.doi.org/10.4067/S0718-19572007000300003

Mujica A. \& M. L. Nava. 2010. Distribución espacial de larvas de crustáceos decápodos planctónicos en canales orientales de la isla Chiloé, Chile. Latin American Journal of Aquactic Research. 38(1): 95-106. http://dx.doi.org/10.3856/vol38issue1-fulltext-9.

Muller-Karger F., R. Varela, R. Thunell, Y. Astor, H. Zhang, R. Luerssen \& C. Hua. 2004. Processes of coastal upwelling and carbon flux in the Cariaco Basin. Deep-Sea Research Part II 51:927-943. https://doi.org/10.1016/j.dsr2.2003.10.010.

Nielsen T. \& F. Anderson. 2003. Phosphorus dynamics during decomposition of mangrove (Rhizophora mangle) leaves in sediments. Journal of Experimental Marine Biology and Ecology. 293: 73-88. http://dx.doi.org/10.1016/j.marenvres.2007.04.007

Palazón, J. 2000. Condiciones hidroquímicas del saco de la Isla de Coche, Venezuela, mayo 1989-mayo 1990. Boletín del Instituto Oceanográfico de Venezuela. 39 (1-2): 55-65.

Palazón J., G. Hernández, J. Hernández \& E. Penoth. 1996. Condiciones hidroquímicas de la Laguna de Las Marites, Isla de Margarita, Venezuela. Abril 1989-mayo 1990. Boletín del Instituto Oceanográfico de Venezuela 35 (1-2): 113-125.

Palazón J. \& E. Penoth. 1998. Condiciones hidroquímicas de las aguas superficiales de la Laguna Boca de Palo, Isla de Margarita, Venezuela. Boletín del Instituto Oceanográfico de Venezuela 37 (1-2): 3-15.

Peck N., J. Peters, R. Diekmann, S. Laakmann \& J. Renz. 2015. Interactive effects of temperature and salinity on population dynamics of the calanoid copepod Acartia tonsa. Journal of Plankton Research 37(1): 197-210. https://doi. org/10.1093/plankt/fbu093.
Pereira C. 2006. Condiciones hidrográficas y estado trófico de la Laguna de Punta de Piedras, Isla de Margarita (febrero 2005-2006). Tesis, Licenciado en Biología Marina, Escuela de Ciencias Aplicadas del Mar. Universidad de Oriente, Venezuela. 80pp.

Pfaff M.C., G.M. Branch, J.L. Fisher, V. Hoffmann, A.G. Ellis \& J.L. Largier. 2015. Delivery of marine larvae to shore requires multiple sequential transport mechanisms. Ecology 96(5): 1399-1410. http://dx.doi.org/10.1890/14-0229.1

Ramírez P. 1996. Lagunas costeras Venezolanas. Porlamar, Venezuela: Universidad de Oriente, Centro Regional de Investigaciones Ambientales. 144pp.

Razouls C., F. de Bovée, J. Kouwenberg \& N. Desreumaux. 2005-2017. Diversity and Geographic Distribution of Marine Planktonic Copepods. Available at <http://copepodes.obs-banyuls.fr/ en> [Accessed April 25 2017].

Reverol Y., J. Delgado, C. López \& L. Sánchez. 2008. Zooplankton community composition in floodplain lakes of Caura river, Venezuela. Boletín del Centro de Investigaciones Biológicas 42(1): 53-72.

Rodrigues M., H. Queiroga, A. Oliveira, V. Brotas \& M.D. Manso. 2016. Climatic and anthropogenic factors driving water quality variability in a shallow coastal lagoon (Aveiro lagoon, Portugal): $1985-2010$ data analysis. AIMS Environmental Science 3(4): 673-696. http://dx.doi.org/10.3934/environsci.2016.4.673

San Martin E., X. Irigoien, R.P. Harris, A. López-Urrutia, M.V. Zubkov \& J.L. Heywood. 2006. Variation in the transfer of energy in marine plankton along a productivity gradient in the Atlantic Ocean. Limnology and Oceanography 51(5): 2084-2091. http://dx.doi.org/10.4319/lo.2006.51.5.2084.

Sendacz, S. 1993. Distribuição geográfica de algúns organismos zooplanctônicos na América do Sul. Acta Limnologica Brasiliensia 6: 31-41.

Sheskin D. 2004. Parametric and nonparametric statistical procedures. Boca Raton: Chapman \& Hall.

Smith, D. L. 1977. A guie to Marine Coastal plancton and marine invertebrate larvae. First Edition. Kendall-Hunt Publishing Company, United States. $161 \mathrm{pp}$.

Souza L.C., C.W.C. Branco, P. Domingos \& S.L.C Bonecker. 2011. Zooplankton of an urban coastal lagoon: composition and association with environmental factors and summer fish kill. Zoologia (Curitiba) 28 (3): 357-364. http://dx.doi. org/10.1590/S1984-46702011000300010.

Suárez-Morales, E., W. J. Reid., M. Life \& F. Fiers. 1996. Catálogo de los copépodos (Crustacea) Continentales de la Península de Yucatán, México. CONABIO y ECOSUR, México. 296p.

Tavares D.C., D.L. Guadagnin, J.F. de Moura, S. Siciliano \& A. Merico. 2015. Environmental and anthropogenic factors structuring waterbird habitats of tropical coastal lagoons: implications for management. Biological Conservation 186: 12-21. https://doi.org/10.1016/j.biocon.2015.02.027

Ter Braak, C.J. \& P. F. Verdonschot. 1995. Canonical correspondence analysis and related multivariate methods in aquatic ecology. Aquatic Sciences 57 (3):255- 289. https://doi.org/10.1007/ BF00877430

Valerio González L., Y. García González, A. Guilarte Bueno, L. Troccoli \& J. López. 2013a. Macroalgas asociadas a neumatóforos del mangle negro Avicennia germinans (L) en laguna El Morro, isla de Margarita, estado Nueva Esparta, Venezuela. Saber, Universidad de Oriente, Venezuela 25 (4): 365-372.

Valerio-González L., Y. García González, S. Levy \& P. Lacabana. 2013b. Inventario florístico de plantas vasculares litorales de la laguna El Morro, isla de Margarita, estado Nueva Esparta, Venezuela. Saber, Universidad de Oriente, Venezuela 25 (2): 151-159.

Vásquez-Suárez A., M. González, O. Díaz \& I. Liñero-Arana. 2010. Variación temporal de la meiofauna en sedimentos del sistema lagunar "laguna de Raya", estado Nueva Esparta, Venezuela. Interciencia: Revista de ciencia y tecnología de América 35(2): 144-150.

Vásquez-Yeomans L., I. Castellanos, E. Suárez-Morales \& R. Gasca. 2012. Variación espacio-temporal de la biomasa de zooplancton en un sistema estuarino del Caribe occidental durante dos ciclos anuales. Revista de Biología Marina y Oceanografía 47(2): 213-225. 
Yáńez-Arancibia A., J.W. Day, P. Sánchez-Gil, J.N. Day, R.R. Lane, D. Zárate-Lomelí \& J. Ramírez-Gordillo. 2014. Ecosystem functioning: The basis for restoration and management of a tropical coastal lagoon, Pacific coast of Mexico. Ecological Engineering 65: 88-100. https://doi.org/10.1016/j. ecoleng.2013.03.007.

Yepez D. 2012. Evaluación de las concentraciones de metales pesados en el caracol Melongena melongena (Linnaeus, 1758) (Mollusca:Gastropoda) y en muestras de sedimento marino como indicadores de contaminación, en la laguna costera del Morro de Porlamar, Isla de Margarita, Edo. Nueva Esparta, Venezuela. Tesis, Licenciado en Biología Marina, Escuela de Ciencias Aplicadas del Mar, Universidad de Oriente, Venezuela. $87 \mathrm{pp}$
Zoppi, E. 1961. Distribución vertical del zooplancton en el Golfo y extremo Este de la Fosa de Cariaco. Boletín del Instituto Oceanográfico de Venezuela 1(1): 219-248.

Zoppi, E. 1974. Comparación de algunas características del plancton entre las lagunas costeras de Tacarigua y Unare, Venezuela. Boletín del Instituto Oceanográfico de Venezuela 13(1-2): 129-146. 